\title{
Behaviors of Bellows-Like Origami Patterned Tubes with Trapezoidal Patterns
}

\author{
Gotou Humihiko $^{1}$, Shibata Katsuya ${ }^{2}$, Kudo Yasuhiro $^{3}$ and Emura Takuro ${ }^{1}$ \\ 1. Department of Civil and Environment Engineering, Faculty of Engineering and Resource Science, Akita University, Akita City \\ 010-8502, Japan \\ 2. Iida Survey Design Company Limited, Kashiwa City 277-0005, Japan \\ 3. TakenakaDoboku Nagoya Branch Co., Ltd., Nagoya City 460-0003, Japan
}

\begin{abstract}
In this paper, we show one of the possibility utilizing typical origami structures for civil engineering fields such as the bridge bearing support. We numerically investigate axial spring constants and buckling behaviors of bellows-like origami tube structures. The bellows-like origami tube structures, which can be folded because of elastic deformations, work as a kind of spring. If the initial heights of the bellows-like origami tubes are less than $90 \%$ of the height of the prismatic tubes without bellows-like folded lines, the spring constants of the bellows-like tubes are very low compared with those of the prismatic tubes. The buckling loads and patterns of the bellows-like tubes vary depending on the initial heights of the tubes.
\end{abstract}

Key words: Origami, foldable cylinder, buckling, CalculiX.

\section{Introduction}

Recently, various kinds of origami structures are studied in various fields like space structure, airplane, architecture, medical device, beverage can and so on. One of the early researches of origami structures is that by Miura [1], who is famous for "Miura fold" [2]. Recent researchers focus on various functions of origami structures [3-6].

There are two types of foldable origami structures: those which can be folded, even if they are assembled from rigid plates (rigid origami [4]), and those which can be folded because of elastic deformations of not only folded lines but also plate parts (bellows-like PCCP (pseudo-cylindrical concave polyhedral) shell [1]). The former structures have widely deployable capabilities, which are useful in space structures and so on, while the latter structures have spring capabilities with also useful potentials in various fields, for example, bridge bearing supports and other spring

Corresponding author: Gotou Humihiko, associate professor, research fields: structural engineering and timber structure. E-mail: gotou@gipc.akita-u.ac.jp. structures utilizing their axial spring and plastic shear deformation capabilities.

In this paper, we numerically investigate axial spring contents and elasto-plastic shear deformation behaviors of the latter deformably foldable bellows-like origami tubes with trapezoidal patterns composed of alternately aligned upright and inverted trapezoids.

We expect that the axial and shear springs of the origami tubes can work as the bridge bearing support for normal time, while their elasto-plastic shear deformation can work as the energy absorption for horizontal earthquake ground motion, such as a kind of functional separation type supports [7].

\section{Models}

The investigated bellows-like tube models can be folded from a thin rectangular plate and composed of alternately aligned upright and inverted trapezoidal plates as shown in Fig. 1.

We use Fortran (g77) programs to make the meshing data for the tubes and use the open source solver 


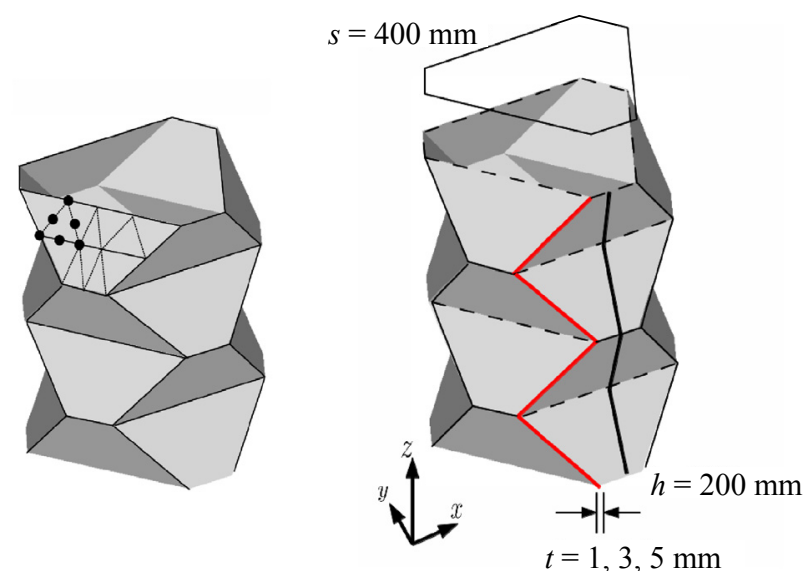

Fig. 1 FEM model of bellows-like tube.

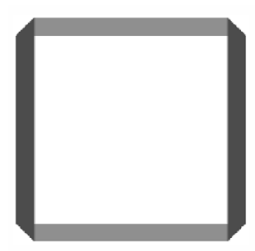

4

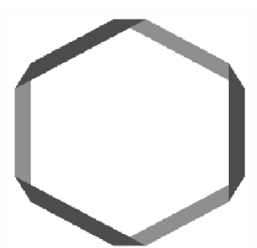

6

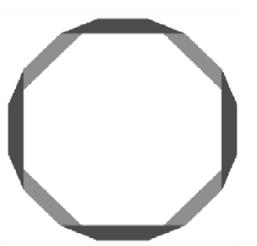

8
Fig. 2 Number of circumferential patterns.

CalculiX to solve the problems. Each trapezoid of the tubes is modeled by $12 \mathrm{~S} 6$ (6-node triangular)elements as shown in Fig. 2.

We specify the number of trapezoidal patterns of the bellows-like tubes in two directions: One is the number of trapezoidal patterns in the circumferential direction and another is the number of trapezoidal patterns in the axial (height) direction. The number of circumferential patterns needs to be even since the trapezoidal patterns are aligned alternately upright and inverted in the circumferential direction.

The bellows-like tubes modeled in this study are folded from the same size thin rectangular plates (circumferential length $s=400 \mathrm{~mm}$, height $h=200$ mm, thickness $t=1,3,5 \mathrm{~mm}$, Young's modulus: $E=$ $206 \mathrm{GPa}$, Poisson's ratio: 0.3 are shown in Table 1) for some possible combinations of the number of circumferential patterns $(4,6,8)$, the number of axial patterns (10) and the initial height ratio (0-1).

The number of circumferential patterns are set to 4 , 6 and 8 as shown in Fig. 2, while the number of axial patterns is fixed to 10 .
The initial height ratio can be from 0 to 1 . When the initial height is 0 , the bellows-like tube is perfectly folded in the plane and when the initial height ratio is 1 , the bellows-like tube become the prismatic tube as shown in Fig. 3. The FEM models of the bellows-like tubes stand vertically along $z$-axis as shown in Fig. 1 . The bottom circumference of the FEM model tube is restrained at all the nodes in $x$-, $y$-, $z$-directions. We investigate two kinds of loading: vertical (axial) compression and horizontal shear for the top circumference. In the case of the vertical compression loading in $z$-direction, displacements in $x$-, $y$-directions at all the nodes of the top circumference are restrained.

In the case of the horizontal shear loading in $x$-direction, displacements in $y$-, $z$-directions and all rotations at all the nodes of the top circumference are restrained. While we estimate that the realistic fabrication approach of the bellows-like tube as the bridge bearing support is press working from a plate, the welded joint joined by press may become weakness. Considering the possibility that the bellows-like tubes are used with slit without welding weakness, we also investigate the FEM models for the

\section{Table 1 Material properties.}

\begin{tabular}{ll}
\hline Property names & Property sizes \\
\hline Thickness $t$ & $1 \mathrm{~mm}, 3 \mathrm{~mm}, 5 \mathrm{~mm}$ \\
Original height $h$ & $200 \mathrm{~mm}$ \\
Circumference $s$ & $400 \mathrm{~mm}$ \\
Young's modulus $E$ & $206 \mathrm{GPa}$ \\
Poisson's ratio & 0.30 \\
\hline
\end{tabular}

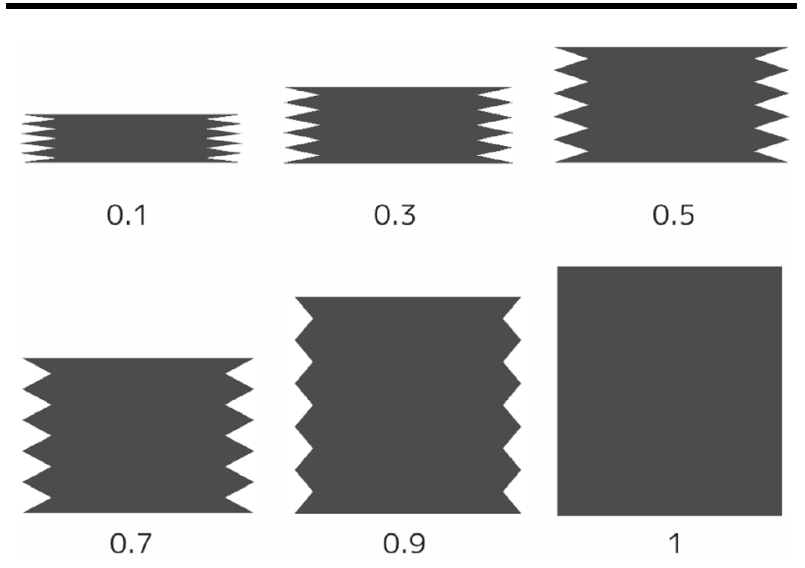

Fig. 3 Initial height ratio of tubes. 
cases with the jag cut line along the inclined sides of the trapezoidal patterns.

\section{Numerical Calculations}

\subsection{Spring Constant}

The relationship between spring constants and initial heights of the bellows-like tubes are shown in Fig. 4 for the case without slit and in Fig. 5 for the case with slit. The spring constant $k$ is derived from the load $P$ and the displacement $\delta$ in the loading direction at the loading point as:

$$
k=\frac{P}{\delta}
$$

In Figs. 4 and 5, the spring constant $k$ is nondimensionalized by the axial spring constant of the circular cylinder tube $k_{\text {circ }}$ given by Young's modulus of the material $E$ as:

$$
k_{\text {circ }}=\frac{E A}{\ell}
$$

where, $A$ is the cross sectional area of the circumference and $\ell$ is the height when the initial height ratio is 1 . In the case of vertical compression, the nondimensionalized spring constants are getting larger along with the increase of the initial heights of the tubes, while in the case of horizontal shear, the spring constants are getting smaller along with the increase of the initial heights of the tubes. In the case without slit, the tubes with the largest spring constants are those whose number of circumferential patterns is 8 , the tubes with the second largest spring constants are those whose number of circumferential patterns is 6 and the tubes with the 3rd largest spring constants are those whose number of circumferential patterns is 4. On the other hand, in the case with slit, the tubes with the largest spring constants are those whose number of circumferential patterns is 4 , the tubes with the second largest spring constants are those whose number of circumferential patterns is 6 and the tubes with the 3rd largest spring constants are those whose number of circumferential patterns is 8 .

\subsection{Cyclic Loading}

In order to investigate the energy absorption capacity of the bellows-like tubes by shear elasto-plastic deformation, we try the shear cyclic loadings in this section. The elasto-plastic curve for the material is given by the generalized Ramberg-Osgood law as follows:

$$
E \varepsilon=\sigma+\alpha\left(\frac{|\sigma|}{\sigma_{0}}\right)^{n-1} \sigma
$$

where, $\varepsilon$ is the logarithmic strain, $\sigma$ is the Cauchy stress, $\sigma_{0}$ is the Cauchy stress, $\alpha$ is the yield offset and $n$ is the exponential factor (Fig. 6). In this study, we use the values $n=8, \alpha=0.5, \sigma_{0}=245 \mathrm{MPa}$ so that the elasto-plastic curve is close to the perfect elasto-plastic one. The shear cyclic load is given by the horizontal loads at all the nodes of the top circumference

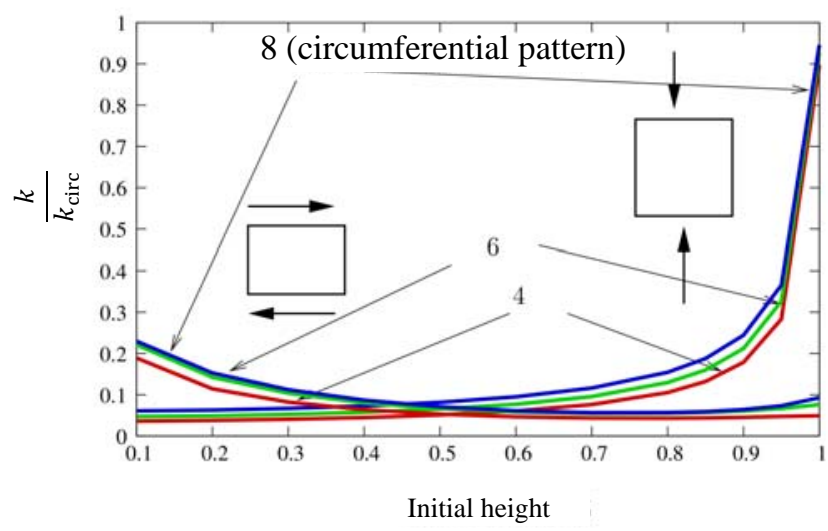

Fig. 4 Spring constant (without slit).

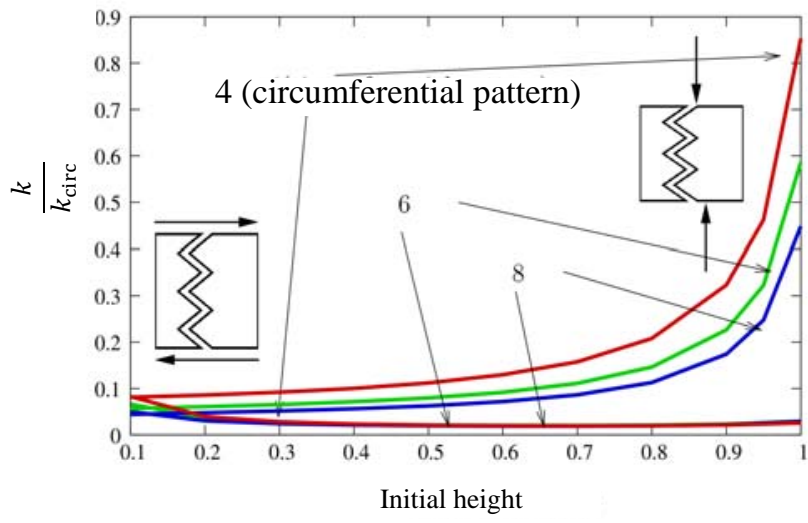

Fig. 5 Spring constant (with slit). 


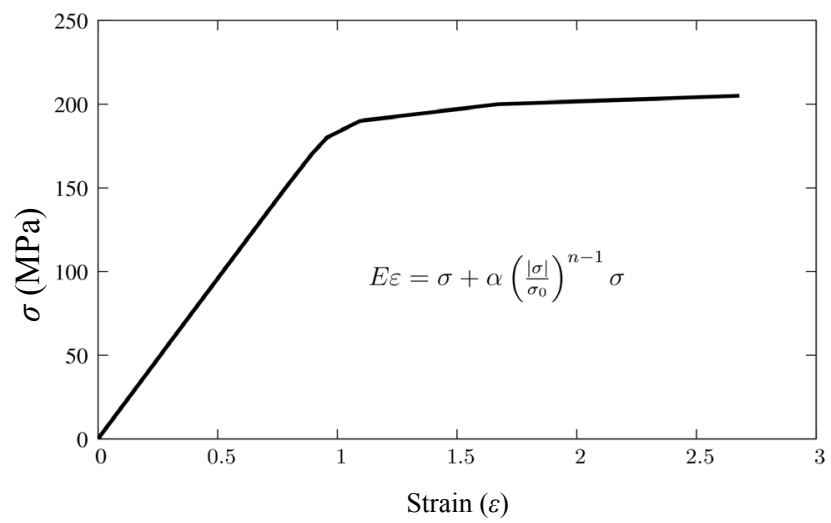

Fig. 6 Elasto-plastic model.

of the tubes. The displacements are calculated by the FEM-solver for given loads, which are incrementally changed. We compare the cases with different initial heights, the cases with different thicknesses and the cases with/without the slit of the bellows-like tubes.

In one of the comparing cases, we set the rough standard turnback load by the lowest load, where the slope of the load-displacement line is getting smaller with plastic deformation in the first loading. As far as the load controlling is possible, we switch from loading to unloading or from unloading to loading around the plus or minus value of the absolute rough standard turnback load for the compared tubes in a comparing case as shown in Fig. 7 so that we can compare the energy absorption capacity of the tubes in the close cyclic loading ranges.

Considering that the larger initial height of the tubes with enough axial stiffness is suitable for the use as bridge bearing supports, the initial height ratios are set to 0.9 in each comparing case except for the case comparing the different initial heights themselves.

\section{Results and Discussion}

\subsection{Comparison of Thickness}

The results comparing the tube thicknesses between $1 \mathrm{~mm}, 3 \mathrm{~mm}$ and $5 \mathrm{~mm}$ are shown in Figs. 8-10 for the cases with the numbers of circumferential patterns 4,6 and 8 , respectively. In these cases with different tube thicknesses, we can not set the rough standard turnback load, since the lowest turnback load with the thinnest tube is thoroughly included within the elastic loading range for other thicker tubes. Hence, the turnback load is set to the load where the load increment step can not be numerically increased in the elasto-plastic area for the each thickness. The absolute value of the minus turnback load is set to the close value around the first plus turnback load, as far as the load controlling is possible. The elastic areas for the

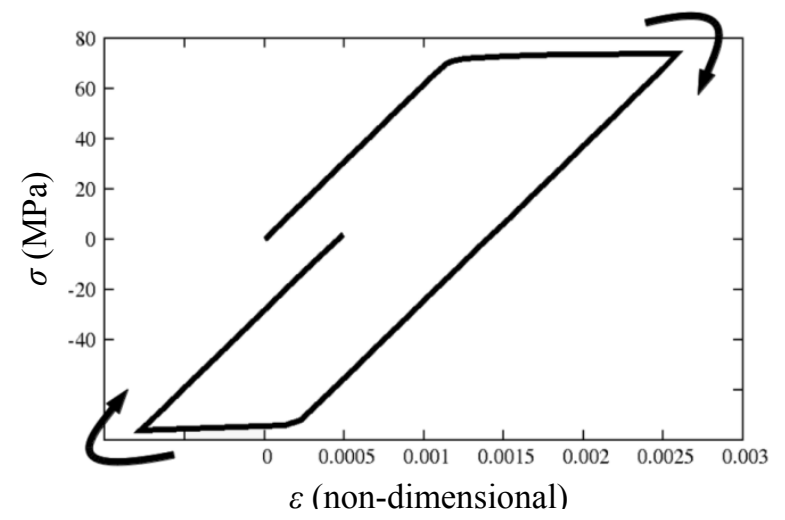

Fig. 7 Turnback loading.

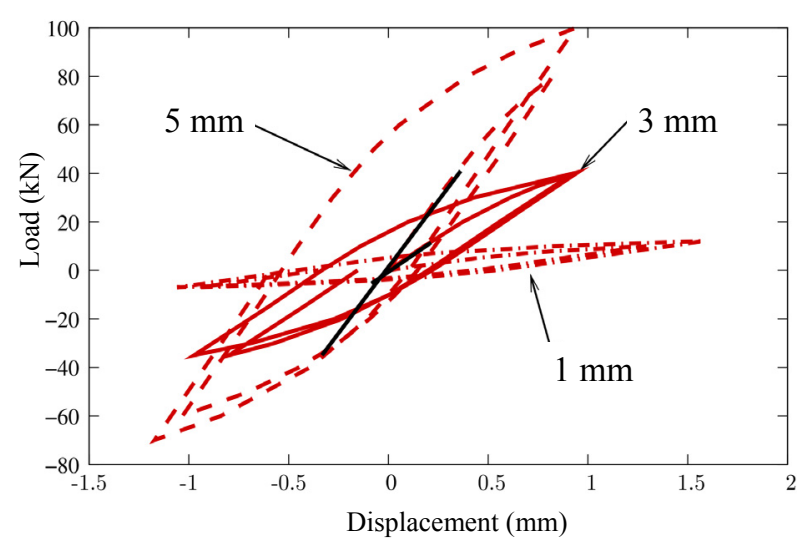

Fig. 8 Comparison of thickness (circumferential: 4).

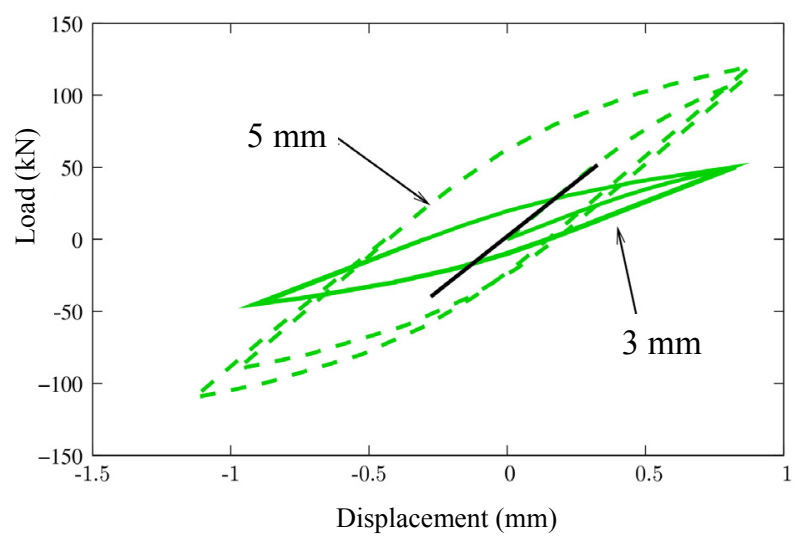

Fig. 9 Comparison of thickness (circumferential: 6 ). 


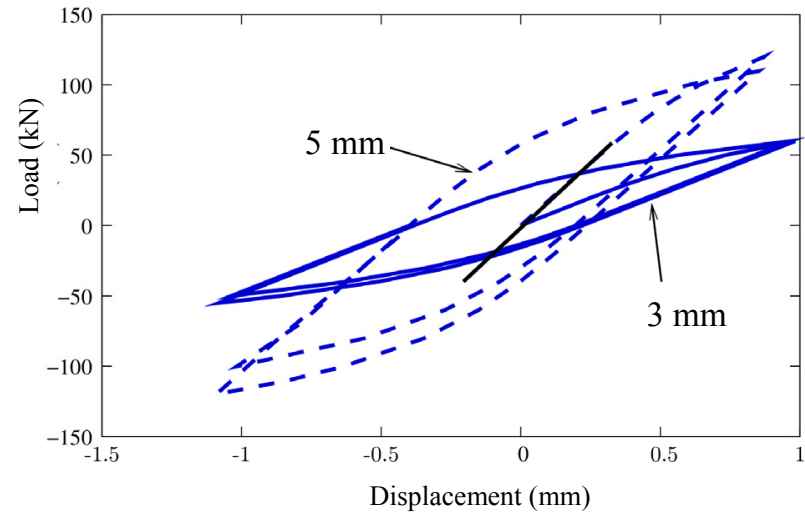

Fig. 10 Comparison of thickness (circumferential: 8).

thickest tubes are shown by solid lines.

The cases with slit investigated for different thicknesses in the same way are shown in Figs. 11-13. The stiffnesses of the tubes with slit are about several tens of percent than that of the tubes without slit.

\subsection{Comparison of Initial Height}

The results changing the initial height ( 0.9 or 0.6 ) for the bellows-like tubes without slit are shown in Figs. 14-16. Unlike in the case of changing the thickness, the turnback loads can be set to the close range for the two initial heights 0.6 and 0.9 .

In all the numbers of circumferential patterns 4,6 and 8 , the results for the two initial heights 0.6 and 0.9 are not so different, as is expected from the results for the spring constants shown in Fig. 4. The results changing the initial height $(0.9$ or 0.6$)$ for the bellows-like tubes with slit are shown in Figs. 17-19. Unlike in the case of the bellows-like tubes without slit, the energy absorptions of the tubes with the initial height 0.9 are getting larger than the initial height 0.6.

While the spring constants for the tubes with slit are not so different between the initial heights 0.6 and 0.9 , as shown in Fig. 5, the energy absorptions by the turnback loading of the tubes with slit for the initial height 0.9 are larger than the height 0.6 .

\subsection{Comparison between Cases with and without Slit}

The results for cases of the bellows-like tubes with and without slit are shown in Figs. 20-22. The tubes

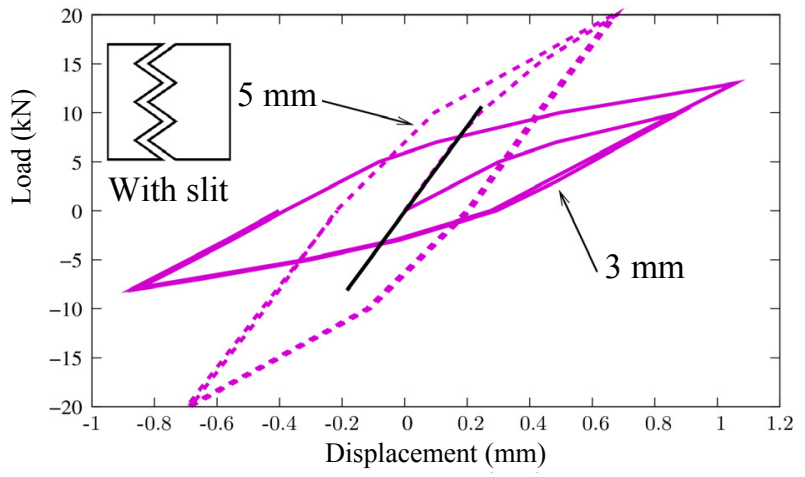

Fig. 11 Comparison of thickness (circumferential: 4, with slit).

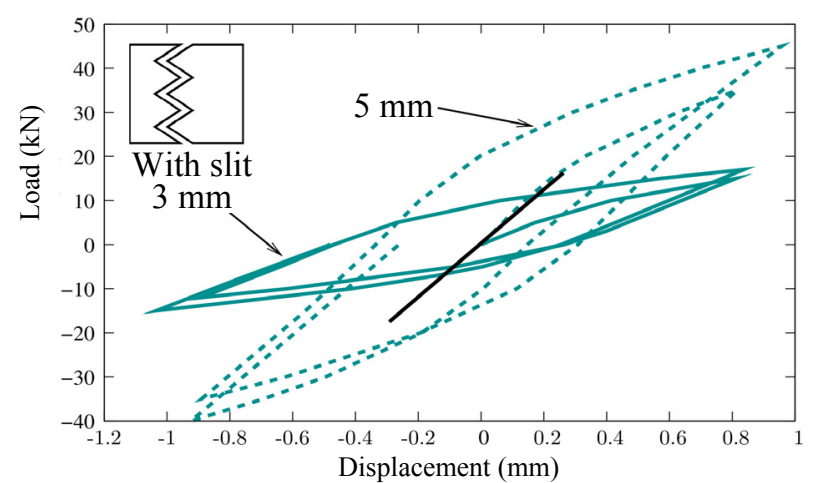

Fig. 12 Comparison of thickness (circumferential: 6, with slit).

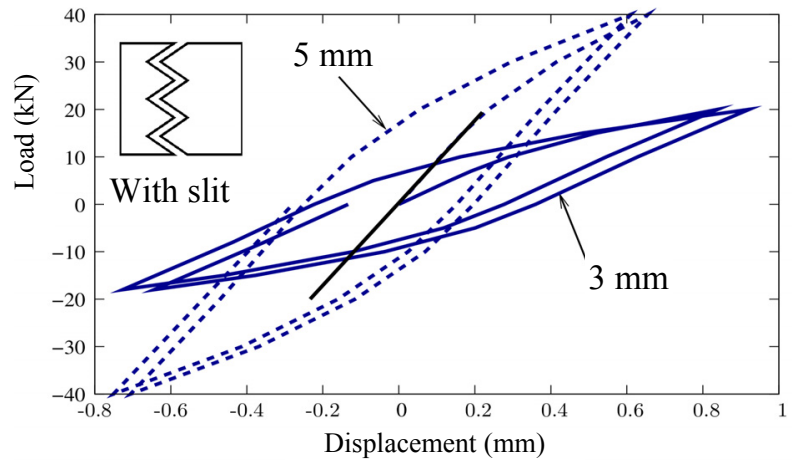

Fig. 13 Comparison of thickness (circumferential: 8, with slit).

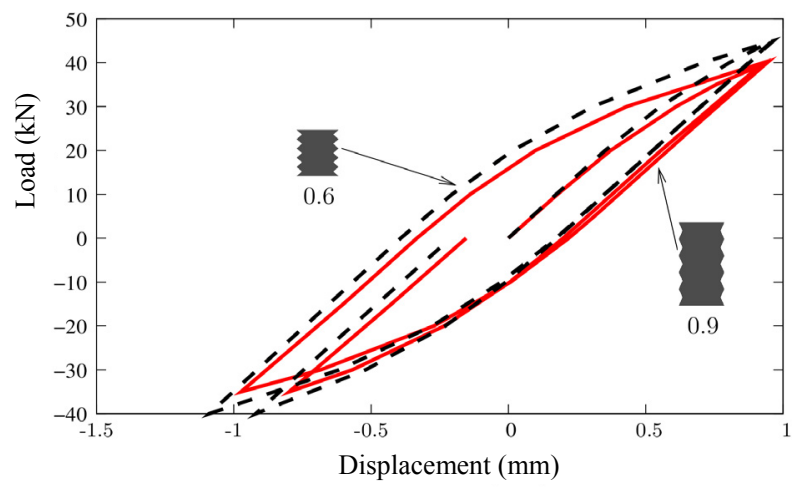

Fig. 14 Comparison of initial height (circumferential: 4). 


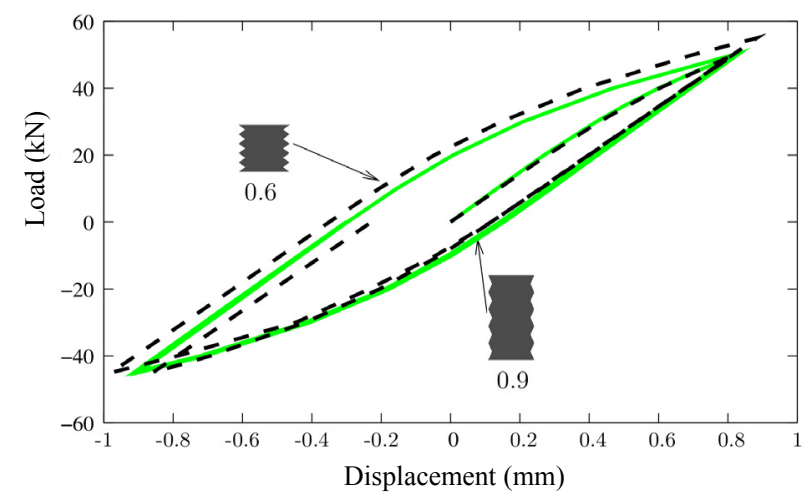

Fig. 15 Comparison of initial height (circumferential: 6).

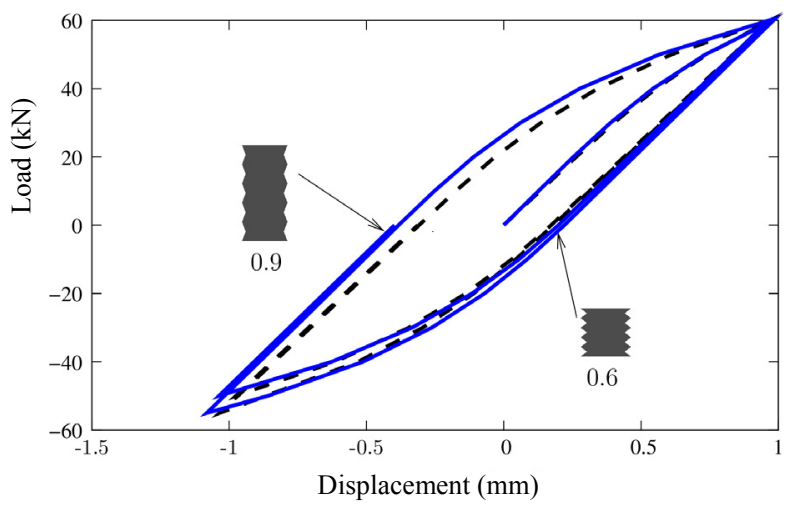

Fig. 16 Comparison of initial height (circumferential: 8).

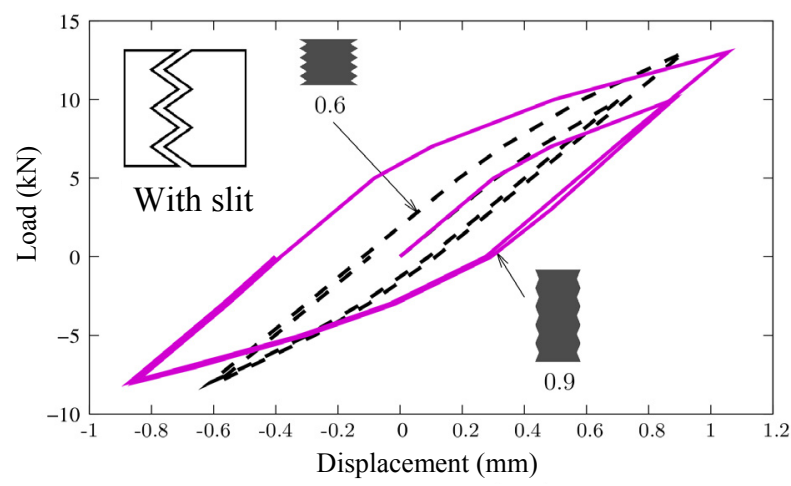

Fig. 17 Comparison of initial height (circumferential: 4, with slit).

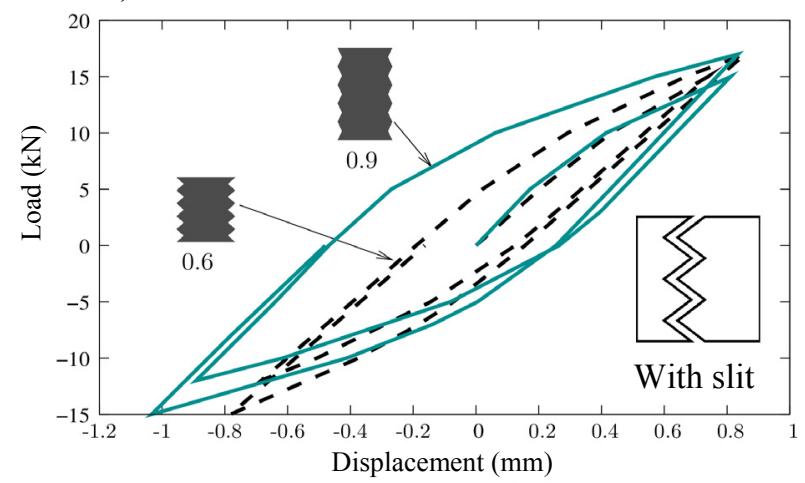

Fig. 18 Comparison of initial height (circumferential: 6, with slit).

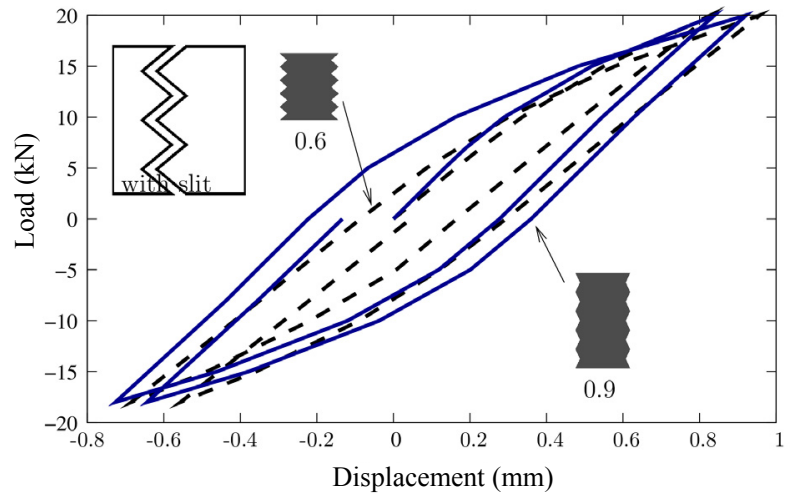

Fig. 19 Comparison of initial height (circumferential: 8, with slit).

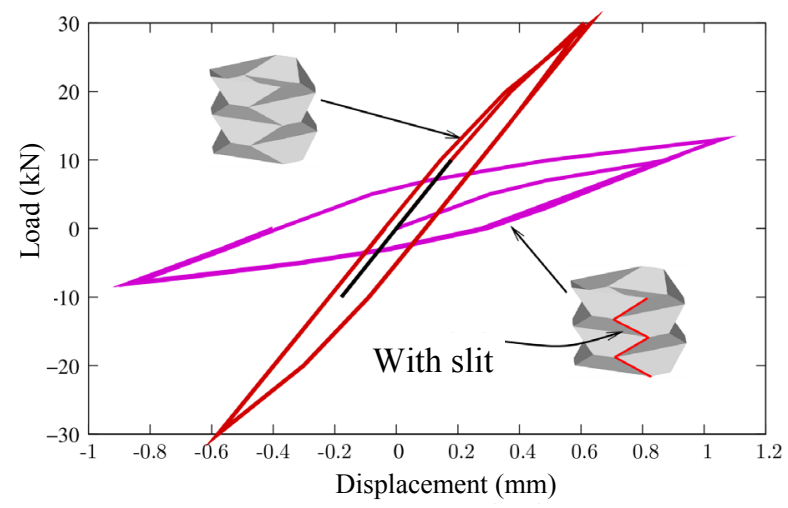

Fig. 20 Comparison between with/without slit (circumferential: 4 ).

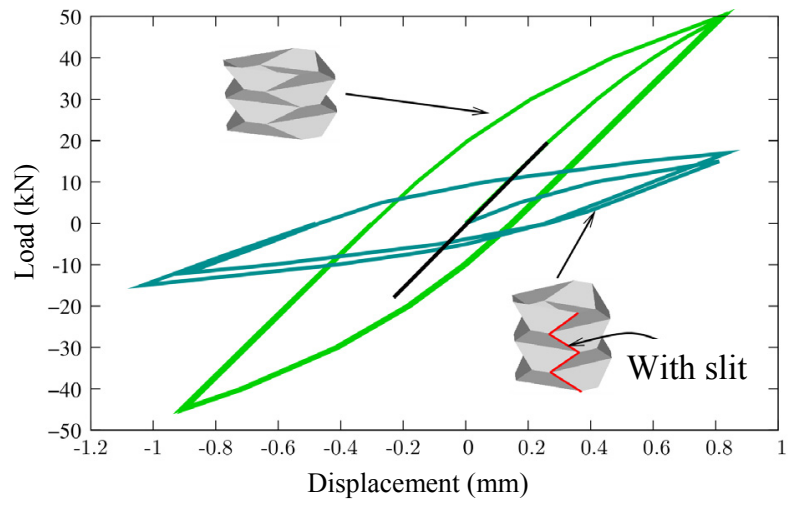

Fig. 21 Comparison between with/without slit (circumferential: 6).

with slit lose the stiffness in much turnback loading range for the tubes with slit, smaller load than those without slit, which remain elastic (shown by solid lines in the figures) within the turnback loading range for the tubes with slit.

\section{Conclusions}

Since the thicker tube remain elastic within the 


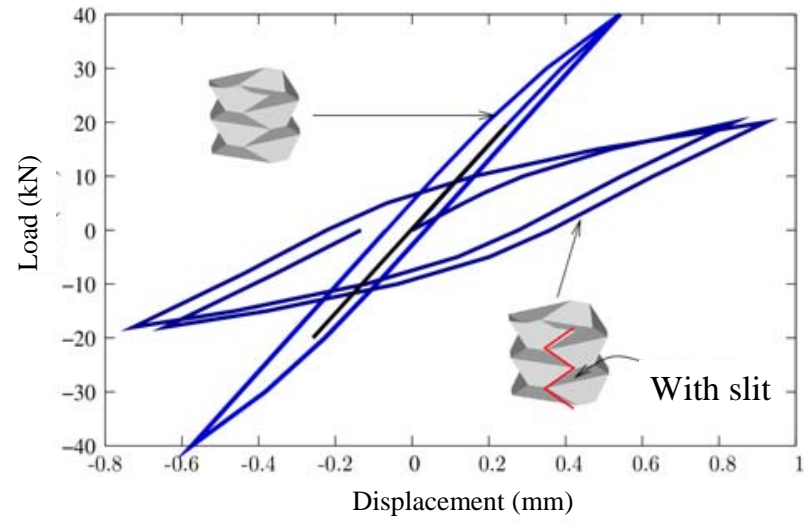

Fig. 22 Comparison between with/without slit (circumferential: 8).

turnback loading range for the thinner tube, the energy absorption capacity of the thinner tube can be larger than the thicker tube in a certain smaller turnback loading range.

The energy absorptions of the tubes with higher initial height are larger than lower initial height for the cases with slit, while such tendency is not observed for the cases without slit.

The tubes which have larger axial spring stiffness and have larger energy absorptions are those with slit and the initial height ratio 0.9 .

These results show the possibility that the tubes, whose spring stiffness and energy absorption are well controlled, are applied to bridge bearing supports.

\section{References}

[1] K. Miura, Proposition of Pseudo-Cylindrical Concave Polyhedral Shells, Report No. 442, Institute of Space and Aeronautical Science, University of Tokyo, 1969.

[2] Y. Nishiyama, Miura folding: Applying origami to space exploration, International Journal of Pure and Applied Mathematics 79 (2) (2012) 269-279.

[3] G.W. Hunta, I. Ario, Twist buckling and the foldable cylinder: An exercise in origami, International Journal of Non-Linear Mechanics 40 (2005) 833-843.

[4] K. Kuribayashi, K. Tsuchiya, Z. You, D. Tomus, M. Umemoto, T. Ito, et al., Self-deployable origami stent grafts as a biomedical application of Ni-rich TiNi shape memory alloy foil, Materials Science and Engineering A 419 (2006) 131-137.

[5] Z. You, Motion structures extend their reach, Materials Today 10 (12) (2007) 52-57.

[6] J. Song, Y. Chen, G. Lu, Axial crushing of thin-walled structures with origami patterns, Original Research Article, Thin-Walled Structures 54 (2012) 65-71.

[7] T. Santo, J. Saito, K. Masuda, Transition and recent knowledge of bridge bearings in Japan, 15th World Conference in Earthquake Engineering, Lisbon, Portugal, 2012. 